\title{
Inteligencia emocional y altas capacidades cognitivas Un estudio empírico
}

\author{
Alejandra Gould Gavidia \\ agould@uji.es \\ Martin Sanchez-Gomez \\ sanchgom@uji.es \\ Edgar Breso Esteve \\ breso@uji.es
}

\section{Resumen}

Hasta la fecha, los estudios que han observado las competencias emocionales en niños con altas capacidades han ofrecido resultados contradictorios (Patty, Brackett, Ferrándiz y Ferrando 2011). Mientras algunos autores indican que los sujetos con altas capacidades presentan mayor inteligencia emocional (IE) que los sujetos normales, otros afirman que tienen mayores problemas de ajuste emocional.

El objetivo de este estudio se centra en comprobar empíricamente si existen diferencias estadísticamente significativas en los niveles de IE entre los sujetos de altas capacidades y los sujetos normotípicos. Para examinar los niveles de IE de los sujetos, se pasó el test de habilidad MEIT a una muestra de 226 alumnos de 8 a 12 años (28 con altas capacidades y 198 normotípicos de la provincia de Castellón).

La prueba T para dos muestras independientes nos indica que el grupo de altas capacidades tiene una media superior estadísticamente significativa en IE total, gestión y percepción de emociones. Sin embargo, las diferencias encontradas en comprensión de emociones no han sido significativas. Nuestros datos indican que el alumnado de altas capacidades puntúa más elevado en IE que los alumnos normales $y$, por tanto, muestra una mayor habilidad para percibir $y$ manejar las emociones. Para futuras investigaciones, sería interesante realizar un estudio longitudinal para ver si estas diferencias varían con la edad.

Palabras clave: altas capacidades cognitivas, inteligencia emocional, evaluación, MEIT. 


\begin{abstract}
Studies that have observed emotional competencies in gifted children with high abilities have offered contradictory results (Patty, Brackett, Ferrándiz \& Ferrando 2011). While some authors indicate that subjects with high abilities have greater emotional intelligence (EI) than normal subjects, others claim that they have greater emotional adjustment problems.

The objective of this study was to empirically prove whether there are statistically significan differences in El levels between subjects with high abilities and normal students. To examine the El levels of the subjects, the MEIT skill test was answered by a sample of 226 students ages 8 to 12 (28 with high abilities and 198 normal students from the Province of Castellón).

Using a T-test for two independent samples, it can be observed that the group with high capacities has a statistically significant higher mean in total $\mathrm{El}$, management and perception of emotions. However, the differences found in understanding emotions has not been statistically significant. Our data indicate that students with high abilities score higher in El than normal students and, therefore, show a greater ability to perceive and manage emotions. For future research, it would be interesting to perform a longitudinal study to see if these differences vary with age.
\end{abstract}

Keywords: high ability students, emotional intelligence, assessment, MEIT.

\title{
Introducción
}

\section{Inteligencia emocional y su evaluación}

El concepto de inteligencia emocional (IE) fue acuñado por Salovey y Mayer en 1990 y alcanzó una gran popularidad en la sociedad gracias a la difusión realizada por Daniel Goleman con su best seller Emotional Intelligence, publicado en 1995. Según el modelo de IE de Mayer y Salovey (1997), la inteligencia emocional es considerada como una habilidad mental que puede ser dividida en cuatro ramas o habilidades diferentes pero interconectadas: percepción emocional, facilitación emocional, comprensión emocional y regulación emocional. Este modelo ha sido reformulado por sus autores en varias ocasiones. En 1999, Mayer, Caruso y Salovey reconocieron que la rama de facilitación emocional y la rama respectiva a la comprensión emocional eran difíciles de diferenciar, ya que tenían un alto nivel de correlación, por lo que aceptaron la posibilidad de combinar ambas habilidades en un solo factor.

La IE forma parte del campo de la inteligencia, correlacionando moderadamente con otras inteligencias, por lo que, al igual que la inteligencia tradicional, las habilidades de IE se van adquiriendo progresivamente con los años y a través de la experiencia (Mayer, Salovey y Caruso 2000; Mayer, Salovey, Caruso y Sitarenios 2001).

Actualmente existen dos enfoques para medir la inteligencia emocional. El primer enfoque mide la IE como una habilidad, evaluando el rendimiento en diversas pruebas emocionales. Aquí se encuentran medidas como la Escala Multifactorial de Inteligencia Emocional (MEIS; Mayer, Caruso y Salovey 1999) y el MSCEIT, el Test de Inteligencia Emocional de Mayer, Salovey y Caruso (2002). Ambas pruebas miden las cuatro ramas de IE mencionadas anteriormente. 
El segundo enfoque considera la IE como un rasgo, considerando una mezcla de disposiciones afectivas, rasgos de personalidad y motivación (Petrides 2009). Miden la inteligencia emocional a través de escalas de autopercepción, como la Escala de Rasgo Meta-Mood (TMMS, Salovey et al. 1995), el Inventario de Cociente Emocional (EQ-i; Bar-On 1997) y el Cuestionario de Inteligencia Emocional Trait (TEIQue, Petrides 2009). Estos cuestionarios no miden los niveles de habilidad de IE en sí mismos, sino más bien la percepción que tiene uno mismo respecto a su habilidad de IE (Mayer, Caruso y Salovey 2016).

\section{Altas capacidades y talentosos}

La alta capacidad intelectual no es un término fácil de definir ya que ha causado controversia con distintas nomenclaturas (alta habilidad, talentoso, superdotación, etc.) y hay una falta de consenso en los aspectos definitorios entre los especialistas (De Cássia, Zeferino, Roama, Zaia y Campos 2016). A modo general, hace referencia a un colectivo diverso que puntúa alto con respecto a la población normal en una o varias áreas cognitivas, evaluado normalmente por su cociente intelectual. Pero además, se tienen en cuenta otros parámetros no psicométricos como la creatividad, la capacidad de aprendizaje superior al resto de sus compañeros, un gran número de estrategias de aprendizaje, una gran curiosidad y motivación (Luque-Parra, Hernández-Díaz y LuqueRojas 2016).

Se sabe poco respecto a las características emocionales y sociales del alumnado con altas capacidades. La investigación en este campo ha recibido poca atención en comparación con la gran cantidad de investigación sobre las características cognitivas de las altas capacidades (Zeidner y Matthews 2017). Patty, Brackett, Ferrándiz y Ferrando (2011) realizaron un análisis de los estudios que han profundizado en las competencias emocionales de este alumnado, obteniendo resultados contradictorios. Mientras algunos autores indican que los alumnos con altas capacidades presentan mayor IE que los sujetos normales, otros afirman que tienen mayores problemas de ajuste emocional.

Los resultados obtenidos hasta la fecha nos indican que los alumnos con altas capacidades presentan algunos problemas. Se ven diferentes al resto de sus compañeros y pueden sentirse aislados, alienados o solos en el colegio, desarrollando problemas de ansiedad, inseguridades y problemas emocionales que no saben cómo afrontar por sí solos (Galbraith y Delisle 2015). Como consecuencia, algunos de estos alumnos desarrollan déficits socioemocionales, caracterizados por un comportamiento social, emocional o interpersonal poco adecuado (Zeidner y Matthews 2017). Además, aunque parezca sorprendente, estos alumnos son especialmente vulnerables al fracaso escolar, mostrando unos resultados inferiores a los esperados según su madurez cognitiva. Parece que podría estar relacionado con algunas variables como la presión y las exigencias que reciben por parte del profesorado y sus padres y por problemas interpersonales y sociales (Peterson 2015). Por tanto, es especialmente importante investigar en este ámbito para conocer las características emocionales del alumnado de altas capacidades y luego diseñar programas curriculares específicos que se adapten a su grado de competencia social y emocional (Zeidner y Matthews 2017). Estos investigadores exponen que realizar programas de educación emocional en los centros educativos podría ser la mejor manera de paliar los problemas del alumnado con altas capacidades, ya que hay evidencia empírica que relaciona la IE con el éxito académico y el bienestar general (Ros Morente, Filella Guiu, Ribes Castells y Pérez Escoda 2017). 
Esta investigación tiene como objetivo comprobar empíricamente si existen diferencias estadísticamente significativas en los niveles de IE entre los sujetos de altas capacidades y los sujetos normotípicos de 8 a 12 años. Siguiendo las investigaciones publicadas hasta el momento en las que se afirma que la inteligencia emocional forma parte del dominio de la inteligencia en general (Mayer, Perkins, Caruso y Salovey 2001), se espera que el alumnado con altas capacidades obtenga puntuaciones superiores en inteligencia emocional a las del alumnado normotípico.

\section{Método}

\section{Muestra}

En este estudio participaron un total de 226 sujetos, 28 de los cuales eran niños y niñas con altas capacidades de la Asociación Castellonense de Apoyo al Superdotado y Talentoso (ACAST) y 198 eran alumnado de primaria de la provincia de Castellón. Para formar parte de la asociación ACAST es imprescindible la acreditación de la condición de alta capacidad mediante el correspondiente informe psicológico firmado por un profesional colegiado. Para alcanzar esta condición, lo habitual es completar varias pruebas de inteligencia y obtener un Cl superior a 120.

La edad de la muestra se comprende entre 8 y 12 años, sie ndo la media del grupo con altas capacidades de 10,15 (DT: 1,16), mientras que la medía del grupo de alumnos normotípicos es de 10,20 años (DT: 1,10). En el grupo de altas capacidades el $58 \%$ de los participantes fueron hombres y, en el caso del grupo normotípico, el 53,5\% fueron hombres. La prueba $\mathrm{T}$ para muestras independientes indicó que no existían diferencias significativas entre el grupo de altas capacidades y el de sujetos normotípicos, ni en edad ni en sexo, con niveles de significación bilateral de 0,062 y 0,074 respectivamente.

Por cuestiones éticas, todos los tutores legales de los participantes dieron su consentimiento informado antes de comenzar del estudio.

\section{Instrumentos}

Para examinar los niveles de inteligencia emocional de los sujetos, se utilizó el MEIT (Sanchez-Gomez y Breso 2019). Este test se caracteriza por medir la IE como una habilidad, evaluando la capacidad de percibir, comprender y gestionar las emociones, así como obtener una puntuación total en inteligencia emocional. La rama de percepción emocional se evalúa mediante tres tipos de tareas a lo largo de un total de 19 pantallas en las que el sujeto debe identificar emociones a través de imágenes, identificar el nivel de activación y el placer que compone una emoción dada y asociar una emoción al rostro que mejor la simbolice. La rama de comprensión emocional se evalúa a lo largo de 7 pantallas en las que se deben realizar tareas relacionadas con la capacidad de entender cómo las emociones simples se agrupan para formar otras más complejas y tareas relacionadas con saber cómo diversos estados emocionales pueden conducir a sentimientos concretos. Por último, la rama de gestión emocional se evalúa mediante una tarea a lo largo de 14 pantallas en las que se invita al usuario a contestar sobre cómo actuaría él en esa situación concreta para solucionar el conflicto emocional de la mejor manera posible.

Decidimos utilizar MEIT por varias razones. En primer lugar, por la facilidad de uso, ya que MEIT es una prueba digital que facilita tanto la recogida como el posterior volcado y análisis de datos. En segundo lugar, por estar adaptado a personas con edades 
comprendidas entre 8 y 15 años. El hecho de que esta herramienta esté totalmente computarizada y permita realizar las diferentes tareas de forma interactiva, como si fuese un juego, añade un componente motivacional especialmente importante en niños. $Y$, por último, porque se trata de una prueba de habilidad basada en el modelo de mayor rigor científico (Salovey y Mayer 1990).

La prueba de fiabilidad de dos mitades en la versión original de MEIT indica una fiabilidad de 0,91. En este estudio la fiabilidad fue de 0,88; se obtuvieron en las diferentes ramas de percepción, comprensión y gestión puntuaciones que oscilan entre 0,77 y 0,89 . La fiabilidad de las tareas individuales osciló entre un mínimo de 0,70 y un máximo de 0,90 .

\section{Procedimiento y análisis de datos}

Al inicio de curso se presentó una propuesta de colaboración para este estudio a ACAST y a diferentes centros de educación. Una vez obtenido el consentimiento informado de los padres, se procedió a examinar los niveles de inteligencia emocional del estudiantado. Se reservaron aulas de informática en sus centros educativos para poder evaluarlos mediante el test de habilidad MEIT. La duración de dicha evaluación fue de una hora.

Tras administrar la prueba, se procedió a realizar el análisis estadístico mediante el programa informático SPSS. Para observar la diferencia entre grupos (altas capacidades y sujetos promedio), se utilizó una prueba $\mathrm{T}$ para dos muestras independientes, donde las variables dependientes fueron las diferentes ramas de la IE (percepción, comprensión y gestión), así como la puntuación total de IE.

\section{Resultados}

\section{Evaluación de inteligencia emocional}

A partir de los datos obtenidos en el test de habilidad MEIT y mediante una prueba T para dos muestras independientes, se observa que el grupo de altas capacidades tiene una media superior estadísticamente significativa en las variables de IE total, gestión y percepción de emociones, con un nivel de significación bilateral de 0,021, 0,048 y 0,031 respectivamente (tabla 1 ).

Sin embargo, las diferencias encontradas en la variable de comprensión de emociones no han sido estadísticamente significativa $(0,35)$, aunque sí superior a los niños y niñas normotípicos, con medias de 101,72 y 98,89 respectivamente, resultando en una diferencia de 2,83 puntos. En la figura 1 se pueden observar las puntuaciones medias de los dos grupos.

Tras observar la distribución de los sujetos en la curva de normalidad, observamos que las puntuaciones en el test siguen un patrón de normalidad según lo esperado. En la variable IE total, los sujetos con altas capacidades presentan una media de 104,11 (DT: 8,9) mientras que el grupo normotípico ostenta una media de 99,12 con una desviación de 12,02 puntos. 
Tabla 1

Resultados MEIT: diferencia de medias de dos muestras independientes

\begin{tabular}{lccc}
\hline & $\mathrm{t}$ & $\mathrm{gl}$ & Sig. (bilateral) \\
\hline IE total & 2,326 & 224 & 0,021 \\
Gestión & 1,987 & 224 & 0,048 \\
Comprensión & 0,936 & 224 & 0,350 \\
Percepción & 2,167 & 224 & 0,031 \\
\hline
\end{tabular}

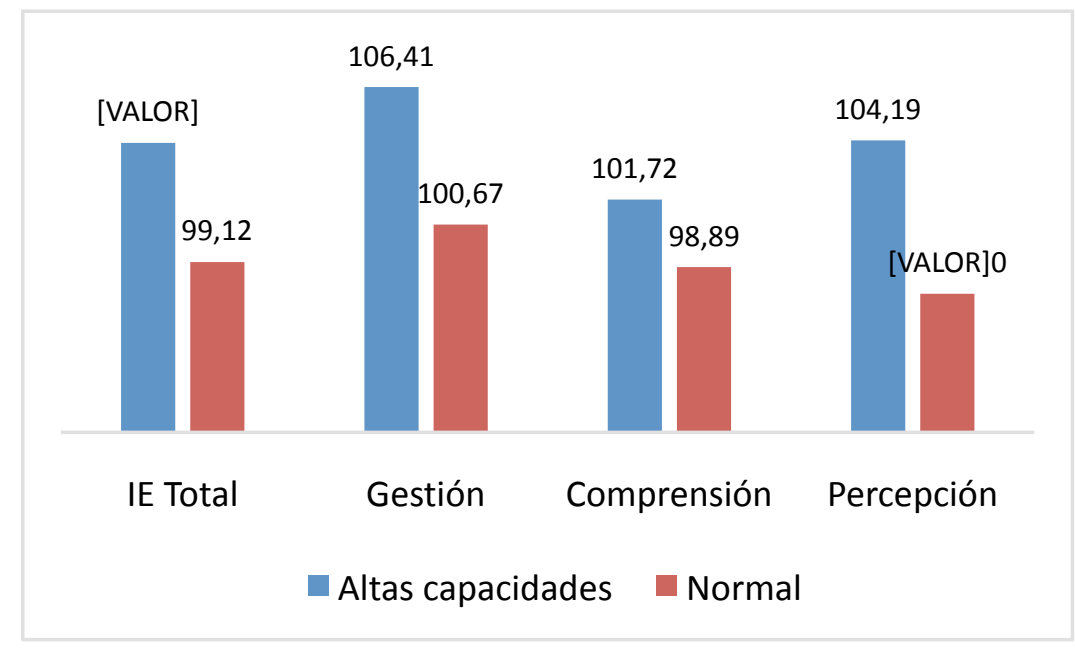

Figura 1. Resultados MEIT: puntuaciones medias de dos muestras.

\section{Discusión y conclusiones}

En este estudio se ha evaluado la capacidad de percibir, comprender y gestionar las emociones de los alumnos normotípicos y de altas capacidades, mediante el test de habilidad MEIT, con el objetivo de comprobar si existen diferencias significativas entre ambos grupos.

La hipótesis de partida queda confirmada mediante los resultados obtenidos, concluyendo que el alumnado con altas capacidades puntúa más elevado en inteligencia emocional que el alumnado normotípico y, además, esta diferencia es estadísticamente significativa. Por tanto, siguiendo la literatura publicada en este ámbito, el alumnado de altas capacidades muestra una mayor habilidad emocional, concretamente en aquellas tareas que tienen que ver con la percepción y la gestión emocional. En cuanto a la compresión de emociones de los participantes con altas capacidades, no ha habido una diferencia significativa respecto al alumnado normotípico, pero sí han obtenido puntuaciones superiores.

Estos resultados concuerdan con otras investigaciones como la de Zeidner, ShaniZinovich, Matthews y Roberts (2005) en la que el alumnado de altas capacidades mostraba una mayor capacidad emocional que los sujetos promedio, según la evaluación realizada mediante el MSCEIT (Mayer, Salovey y Caruso 2002). Asimismo, los hallazgos encontrados en este estudio coinciden con la visión de Mayer, Perkins, 
Caruso y Salovey (2001), ya que los alumnos de altas capacidades no solo puntúan alto en $\mathrm{Cl}$ sino también en las tres ramas de la $\mathrm{IE}$, mientras que el alumnado normotípico tiene un rango de $\mathrm{Cl}$ e IE promedio.

Dentro de los centros educativos, se debe velar por el desarrollo personal, intelectual, social y emocional de todo el alumnado, sin olvidarse del alumnado de altas capacidades (Luque-Parra, Hernández-Díaz y Luque-Rojas 2016). El nivel de inteligencia emocional en niños y niñas con altas capacidades debe ser un elemento que se debe considerar, ya que forma parte de sus potencialidades y tienen que ser incluidas en la respuesta educativa de sus necesidades específicas. Por otra parte, algunos alumnos con altas capacidades presentan déficits socioemocionales, inseguridades y ansiedad entre otros problemas enunciados, como ha sido mencionado previamente. Debido a esto y siguiendo a Zeidner y Matthews (2017), es especialmente beneficioso para este colectivo trabajar la inteligencia emocional. El orientador educativo puede utilizar la inteligencia emocional como marco conceptual para diseñar planes de prevención e intervención útiles en el centro escolar que beneficien tanto al alu mando normotípico como al alumnado de altas capacidades.

Cabe señalar las limitaciones de este estudio, empezando por el tamaño de la muestra. Como se ha podido observar, el número de sujetos de altas capacidades (28 sujetos) es realmente reducido en comparación con el número de sujetos normotípicos (198 sujetos). Sin embargo, se debe tener en cuenta que la distribución de población entre estos dos grupos también es desigual en la sociedad, donde se estima que un $10 \%$ de la población podría ser de altas capacidades (AEST s. f.). Según las estadísticas de EDUCAbase del Ministerio de Educación, Cultura y Deporte (s. f.), en la Comunidad Valenciana el alumnado total matriculado en curso 2016-2017 en educación primaria fue de 318533 , entre los cuales solo 684 sujetos tenían diagnóstico de altas capacidades, lo que representa un $0,21 \%$ de la población. En nuestro estudio las altas capacidades corresponden a un $12,39 \%$ del total de la muestra, por tanto consideramos que, aunque la muestra es reducida, es suficiente para encontrar relaciones significativas. Por otra parte, la falta de consenso en investigaciones previas resulta en una limitación del estudio, ya que carece de bases sólid as de las que partir en nuestra investigación.

En investigaciones posteriores sería conveniente replicar este estudio con otra muestra más amplia y observar los valores del factor de comprensión de emociones, ya que podría alcanzar la significación, como el resto de los factores de IE. Otras investigaciones en esta línea podrían resultar interesantes, como realizar un estudio longitudinal para ver si las diferencias entre altas capacidades y normotípicos varían con la edad y la experiencia, ya que la inteligencia emocional se va adquiriendo progresivamente durante las etapas evolutivas.

\section{Referencias bibliográficas}

Asociación Española de Superdotados y con Talento para niños, adolescentes y adultos. Sin fecha. "Altas capacidades o superdotados». Recuperado de: https://www.aest.es/altas-capacidades-intelectuales-o-superdotacion/ [Consultado el 4 de agosto de 2019].

Baron, Robert. 1997. The BarOn Emotional Quotient Inventory (BarOn EQ-i). Toronto, ON: Multi-Health Systems Inc.

De Cássia, Tatiana N., Marcela G. Zeferino, Rauni Jandé Roama, Priscila Zaia y Carolina Rosa Campos. 2016. «Investigación de la Eficacia de una Escala de 
Evaluación de altas habilidades. Versión profesor». REXE. Revista de Estudios y Experiencias en Educación 15(29): 83-94.

Galbraith, Judy y James R. Delisle. 2015. When gifted kids don't have all the answers: How to meet their social and emotional needs. Minneapolis, MN: Free Spirit Publishing.

Goleman, Daniel. 1995. Inteligencia Emocional. Barcelona: Kairós.

Luque-Parra, Diego Jesús, Ricardo Hernández-Díaz y María Jesús Luque-Rojas. 2016. «Aspectos psicoeducativos en la evaluación del alumnado con altas capacidades intelectuales: Análisis de un caso». Summa Psicológica UST 13(1): 77-88.

Mayer, John. D. y Peter Salovey. 1997. "What is emotional intelligence?» En Emotional development and emotional intelligence: educational implications, ed. Peter Salovey y David Sluyter (3-31). New York: Basic Books.

Mayer, John D., David R. Caruso y Peter Salovey. 1999. «Emotional intelligence meets traditional standards for an intelligence». Intelligence 27(4): 267-298.

-. 2016. "The ability model of emotional intelligence: Principles and updates». Emotion Review 8(4): 290-300.

Mayer, John D., Donna M. Perkins, David R. Caruso y Peter Salovey. 2001. «Emotional intelligence and giftedness». Roeper Review 23: 131-137.

Mayer, John D., Peter Salovey y David R. Caruso. 2000. "Emotional intelligence as zeitgeist, as personality, and as a mental ability». En The handbook of emotional intelligence: Theory, development, assessment, and application at home, school, and in the workplace, ed. Reuven Bar-On y James D. A. Parker (92-117). San Francisco: Jossey-Bass.

-. 2002. Mayer-Salovey-Caruso Emotional Intelligence Test (MSCEIT) user's manual. Toronto: Multi-Health Systems.

Mayer, John D., Peter Salovey, David R. Caruso y Gill Sitarenios. 2001. «Emotional intelligence as a standard intelligence». Emotion 1, 232-242.

Ministerio de Educación, Cultura y Deporte. Sin fecha. EDUCAbase. Recuperado de: http://estadisticas.mecd.gob.es/EducaJaxiPx/Datos.htm?path=/Educacion/Alum nado/Matriculado/Series18/SeriesAlumnado//I0/\&file=Alumnado8.px\&type=pcax is [Consultado el 4 de agosto de 2019].

Patty, Janet, Marc Brackett, Carmen Ferrándiz y Mercedes Ferrando. 2011. "¿Por qué y cómo mejorar la inteligencia emocional de los alumnos superdotados?». Revista Electrónica Interuniversitaria de Formación del Profesorado 14(3): 145156.

Peterson, Jean Sunde. 2015. «School counselors and gifted kids: Respecting both cognitive and affective». Journal of Counseling \& Development 93(2): 153-162.

Petrides, Konstantinos V. 2009. «Psychometric properties of the Trait Emotional Intelligence Questionnaire». En Advances in the assessment of emotional intelligence, ed. Con Stough, Donald H. Saklofske y James D. A. Parker (2133). New York: Springer.

Ros Morente, Agnes, Gemma Filella Guiu, Ramona Ribes Castells y Núria Pérez Escoda. 2017. "Análisis de la relación entre competencias emocionales, autoestima, clima de aula, rendimiento académico y nivel de bienestar en educación primaria». Revista Española de Orientación y Psicopedagogía 28(1): 8-18.

Salovey, Peter y John D. Mayer. 1990. «Emotional intelligence». Imagination, Cognition and Personality 9: 185-211.

Salovey, Peter, John D. Mayer, Susan Lee Goldman, Carolyn Turvey y Tibor P. Palfai. 1995. «Emotional attention, clarity, and repair: Exploring emotional intelligence using the Trait Meta-Mood Scale». En Emotion, disclosure, \& health, ed. James W. Pennebaker (125-154). Washington, DC: American Psychological Association. http://dx.doi.org/10.1037/10182-006 
Sanchez-Gomez, Martin y Edgar Breso. 2019. "The Mobile Emotional Intelligence Test (MEIT): An Ability Test to Assess Emotional Intelligence at Work». Sustainability 11(3): 827. https://doi.org/10.3390/su11030827

Zeidner, Moshe y Gerald Matthews. 2017. «Emotional intelligence in gifted students». Gifted Education International 33(2): 163-182.

Zeidner, Moshe, Inbal Shani-Zinovich, Gerald Matthews y Richard D. Roberts. 2005. «Assessing emotional intelligence in gifted and non-gifted high school students: Outcomes depend on the measure». Intelligence 33(4): 369-391. 